\title{
Surfaces of finite III-type in the Euclidean 3-space
}

\author{
HASSAN AL-ZOUBI ${ }^{1}$, FARHAN ABDEL-FATTAH ${ }^{2}$, MUTAZ AL-SABBAGH ${ }^{3}$ \\ ${ }^{1}$ Department of Mathematics, Al-Zaytoonah University of Jordan P.O. Box 130, Amman 11733, \\ JORDAN \\ ${ }^{2}$ Department of Cyber Security, Al-Zaytoonah University of Jordan P.O. Box 130, Amman 11733, \\ JORDAN \\ ${ }^{3}$ Department of Basic Engineering, Imam Abdulrahman bin Faisal University \\ SAUDI ARAIBIA
}

\begin{abstract}
In this paper, we firstly investigate some relations regarding the first and the second Laplace operators corresponding to the third fundamental form III of a surface in the Euclidean space $E^{3}$. Then, we introduce the finite Chen type surfaces of revolution with respect to the third fundamental form which Gauss curvature never vanishes.
\end{abstract}

Key-Words: - Surfaces in the Euclidean 3-space, Surfaces of finite Chen-type, Laplace operator, Surfaces of revolution.

Received: July 30, 2021. Revised: December 7, 2021. Accepted: December 20, 2021. Published: December 30, 2021.

\section{Introduction}

In 1983 B. Y. Chen introduces the notion of Euclidean immersions of finite type, [16], and from that time on the research into surfaces of finite type has grown up as one can see in the literature in this area. Many results in this field have been collected in [17]. In this respect, the first-named author with S. Stamatakis has given in [23] a new generalization to this area of study by giving a similar definition of surfaces of finite type.

Let $\boldsymbol{x}$ be an isometric immersion of a surface $M$ in the 3-dimensional Euclidean space $E^{3}$. We represent by $\Delta^{J}$ the Laplacian operator of $M$ acting on the space of smooth functions $\mathrm{C}^{\infty}(M)$. Then $M$ is said to be of finite $J$-type, $J=I, I I, I I I$, if the position vector $\boldsymbol{x}$ of $M$ can be decomposed as a finite sum of eigenvectors of $\Delta^{J}$ of $M$, that is

where

$$
\boldsymbol{x}=\boldsymbol{x}_{\boldsymbol{0}}+\boldsymbol{x}_{1}+\boldsymbol{x}_{2}+\ldots+\boldsymbol{x}_{k}
$$

$$
\Delta^{J} \boldsymbol{x}_{\mathrm{i}}=\lambda_{i} \boldsymbol{x}_{i}, i=1, \ldots, k,
$$

$\boldsymbol{x}_{\boldsymbol{0}}$ is a fixed vector and $\lambda_{1}, \lambda_{2}, \ldots, \lambda_{k}$ are eigenvalues of the operator $\Delta^{J}$.

Surfaces of finite type in $E^{3}$ regarding the second fundamental form were investigated for some important classes of surfaces. More precisely, the class of ruled surfaces was studied in [7], while in [3], H. AL-Zoubi studied tubular surfaces in $E^{3}$. Other classes such as translation surfaces, Quadric surfaces, surfaces of revolution, helicoidal surfaces, cyclides of Dupin, and spiral surfaces, the classification of its finite II-type surfaces still unknown. According to the third fundamental form, ruled surfaces in [4], tubes in [5], and quadric surfaces [6] are the only classes were investigated in $E^{3}$.

This type of study can be also extended to any smooth map, not necessary for the position vector of the surface, for example, the Gauss map of a surface. Regarding this see $[8,9]$.

Another generalization to the above, one can study surfaces in $E^{3}$ whose position vector $\boldsymbol{x}$ satisfies the following condition

$$
\Delta^{J} \boldsymbol{x}=\mathrm{A} \boldsymbol{x}, \quad J=I, I I, I I I,
$$

where $\mathrm{A} \in \mathfrak{R}^{3 \times 3}$.

Related to this, in [26] it was proved that the spheres and the catenoids are the only surfaces of revolution satisfying the above equation. Similarly, in [1] it was shown that helicoids and spheres are the only quadric surfaces in $E^{3}$ that satisfy (2). Next, in [2] condition (2) was studied for the class of translation surfaces. In fact, authored ascertained that Scherk's surface is the only translation surface in the Euclidean 3-space that satisfies (2), finally, in [24] the authors studied the class of translation surfaces in $\mathrm{Sol}_{3}$ satisfying (2). Surfaces satisfying condition (2) are said to be of coordinate finite $J$ type.

Another interesting study is to find surfaces in $E^{3}$ whose Gauss map $N$ satisfies the relation (2) that is 


$$
\Delta^{J} N=\mathrm{A} N, \quad J=I, I I, I I I,
$$

For this problem, readers can be referred to [10, $11,13,18,20]$.

Interesting research also one can follow the idea in [21] by defining the first and second Laplace operator using the definition of the fractional vector operators.

In order to achieve our goal, we briefly introduce a formula for $\Delta^{I I I} \boldsymbol{x}$ and $\Delta^{I I I} \boldsymbol{N}$ by using tensors calculations. Further, in the last section, we contribute to the solution of our main result.

\section{Fundamentals}

We consider a smooth surface $M$ in $E^{3}$ given by a patch $\boldsymbol{x}=\boldsymbol{x}\left(u^{1}, u^{2}\right)$ on a region $\mathrm{U}:=(a, b) \times \mathfrak{R}$ of $\mathfrak{R}^{2}$ in which does not contain parabolic points. We denote by

$$
I=g_{i j} d u^{i} d u^{j}, \quad I I=b_{i j} d u^{i} d u^{j}, \quad I I I=e_{i j} d u^{i} d u^{j}
$$

the three fundamental forms of $M$. For any two differentiable functions $f\left(u^{1}, u^{2}\right)$ and $g\left(u^{1}, u^{2}\right)$ on $M$, the first differential parameter of Laplace regarding the fundamental form $J$ is defined by [12]

$$
\nabla^{J}(f, g):=d^{i j} f_{/ i} g_{/ j}
$$

where $f_{f i}:=\frac{\partial f}{\partial u^{i}}$ and $\left(d^{i j}\right)$ denotes the inverse tensor of $\left(g_{i j}\right),\left(b_{i j}\right)$ and $\left(e_{i j}\right)$ for $J=I, I I$ and $I I I$ respectively.

We first prove the following relations:

$$
\begin{aligned}
& \nabla^{I}(f, \boldsymbol{x})+\nabla^{I I}(f, \boldsymbol{N})=0, \\
& \nabla^{I I}(f, \boldsymbol{x})+\nabla^{I I I}(f, \boldsymbol{N})=0 .
\end{aligned}
$$

For the proof of (5) we use (3) and the Weingarten equations

$$
\boldsymbol{N}_{/ j}=-e_{j k} b^{k m} \boldsymbol{x}_{/ m}=-b_{j k} g^{k m} \boldsymbol{x}_{/ m}
$$

to obtain

$$
\begin{aligned}
\nabla^{I I}(f, \boldsymbol{N}) & =b^{i j} f_{/ i} \boldsymbol{N}_{/ j}=-b^{i j} f_{/ i} b_{j k} g^{k m} \boldsymbol{x}_{/ m} \\
= & -g^{i m} f_{/ i} \boldsymbol{x}_{/ m}=-\nabla^{I}(f, \boldsymbol{x}),
\end{aligned}
$$

being (4). We have similarly

which is (5).
The second Laplace operator according to the fundamental form $J=I, I I, I I I$ of $M$ is defined by [10]

$$
\Delta^{J} f:=-d^{i j} \nabla_{i}^{J} f_{/ j}
$$

where $f$ is a sufficiently differentiable function, $\nabla_{i}^{J}$ is the covariant derivative in the $u^{i}$ direction with respect to the fundamental form $J$ [12]. For $J=I I I$ we have

$$
\Delta^{I I I} f=-e^{i j} \nabla_{i}^{I I I} f_{j j}
$$

We now compute $\Delta^{I I I} \boldsymbol{x}$ and $\Delta^{I I I} \boldsymbol{N}$. From (7) and the equations [19, p.128]

$$
\nabla_{j}^{I I I} \boldsymbol{x}_{/ i}=-b^{k m} \nabla_{m}^{I} b_{i j} \boldsymbol{x}_{/ k}+b_{i j} \boldsymbol{N}
$$

we get

$$
\Delta^{I I I} \boldsymbol{x}=e^{i j} b^{k m} \nabla_{m}^{I} b_{i j} \boldsymbol{x}_{/ k}-e^{i j} b_{i j} N .
$$

Denote by

$$
\Lambda_{i j}{ }^{k}:=\frac{1}{2} e^{k m}\left(-e_{i j / m}+e_{i m / j}+e_{j m / i}\right),
$$

the Christoffel symbols of the second kind regarding the third fundamental form. We put

$$
\begin{gathered}
T_{i j}{ }^{k}:=\Gamma_{i j}{ }^{k}-\Pi_{i j}{ }^{k} . \\
\tilde{T}_{i j}^{k}:=\Lambda_{i j}{ }^{k}-\Pi_{i j}{ }^{k} .
\end{gathered}
$$

It is known that $[19, \mathrm{p} .22]$

$$
\begin{aligned}
T_{i j}{ }^{k} & =-\frac{1}{2} b^{k m} \nabla_{m}^{I} b_{i j}, \\
\tilde{T}_{i j}^{k} & =-\frac{1}{2} b^{k m} \nabla_{m}^{I I I} b_{i j},
\end{aligned}
$$

and

$$
T_{i j}^{k}+\tilde{T}_{i j}^{k}=0 .
$$

Besides, using Ricci' s Lemma

$$
\nabla_{j}^{I I I} e^{i k}=0
$$

and the formula

$$
\mathrm{R}=\frac{2 H}{K}=e^{i k} b_{i k},
$$

where $K$ is the Gauss curvature and $H$ is the mean curvature of $M$ respectively we have

$$
R_{/ m}=\nabla_{m}^{I I I}\left(e^{i k} b_{i k}\right)=e^{i k} \nabla_{m}^{I I I} b_{i k}
$$

From (9), (10), (11) and (13) we find 


$$
\begin{aligned}
& e^{i j} b^{k m} \nabla_{m}^{I} b_{i j}=-2 e^{i j} T_{i j}{ }^{k}=2 e^{i j} \tilde{T}_{i j}^{k} \\
& =-e^{i j} b^{k m} \nabla_{m}^{I I I} b_{i j}=-b^{k m} R_{/ m}
\end{aligned}
$$

and so

$$
e^{i j} b^{k m} \nabla_{m}^{I} b_{i j} \boldsymbol{x}_{/ k}=-b^{k m} R_{/ m} \boldsymbol{x}_{/ k}=-\nabla^{I I}(R, \boldsymbol{x}) .
$$

By combining (8), (12), and (14) we obtain [23]

$$
\Delta^{I I I} \boldsymbol{x}=-\nabla^{I I}(\mathrm{R}, \boldsymbol{x})-R N .
$$

Finally, using (5) we arrive at

$$
\Delta^{I I I} \boldsymbol{x}=\nabla^{I I I}(R, N)-R N .
$$

From (15) we obtain the following theorems [23]

Theorem 1. A surface $M$ in $E^{3}$ is of null III-type 1 if and only if $M$ is minimal.

Theorem 2. A surface $M$ in $E^{3}$ is of III-type 1 if and only if $M$ is part of a sphere.

For the normal vector $\boldsymbol{N}$ we have

$$
\nabla_{k}^{I I I} N_{/ i}=-e_{i k} N
$$

we have

$$
\Delta^{I I I} \boldsymbol{N}=-e^{i k} \nabla_{k}^{I I I} \boldsymbol{N}_{/ i}=e^{i k} e_{i k} \boldsymbol{N},
$$

so that we conclude

$$
\Delta^{I I I} N=2 N .
$$

From the last equation, it can be seen that the Gauss map of every surface $M$ in $E^{3}$ is of finite IIItype 1 , the corresponding eigenvalue is 2 .

Now we prove some relations according to the third fundamental form of $M$.

For any differentiable function $f\left(u^{1}, u^{2}\right)$ it can be easily shown that

$$
\begin{aligned}
& \Delta^{I I I}(f \boldsymbol{x})=\left(\Delta^{I I I} f\right) \boldsymbol{x}+f \Delta^{I I I} \boldsymbol{x}-2 \nabla^{I I I}(f, \boldsymbol{x}) \\
= & \left(\Delta^{I I I} f\right) \boldsymbol{x}+f \nabla^{I I I}(R, N)-f R \boldsymbol{N}-2 \nabla^{I I I}(f, \boldsymbol{x})
\end{aligned}
$$

Similarly

$$
\begin{gathered}
\Delta^{I I I}(f \boldsymbol{N})=\left(\Delta^{I I I} f\right) \boldsymbol{N}+f \Delta^{I I I} \boldsymbol{N}-2 \nabla^{I I I}(f, \boldsymbol{N}) \\
=\left(\Delta^{I I I} f\right) \boldsymbol{N}+2 f \boldsymbol{N}+2 \nabla^{I I}(f, \boldsymbol{x})
\end{gathered}
$$

Denote by $W=-<\boldsymbol{x}, \boldsymbol{N}>$ the support function of $M$, where <,> is the Euclidean inner product. Applying relation (7) for the function $W$, it can be easily verified that

$$
\begin{gathered}
\Delta^{I I I} W=-e^{i k} \nabla_{k}^{I I I} W_{/ i}=e^{i k} \nabla_{k}^{I I I}<\boldsymbol{x}, \boldsymbol{N}>_{/ i} \\
=<e^{i k} \nabla_{k}^{I I I} \boldsymbol{x}_{/ i}, \boldsymbol{N}>+<\boldsymbol{x}, e^{i k} \nabla_{k}^{I I I} \boldsymbol{N}_{/ i}> \\
+2 e^{i k}<\boldsymbol{x}_{/ i}, \boldsymbol{N}_{/ k}>=-<\Delta^{I I I} \boldsymbol{x}, \boldsymbol{N}> \\
-<\boldsymbol{x}, \Delta^{I I I} \boldsymbol{N}>-2 e^{i k}<\boldsymbol{x}_{/ i}, b_{k t} g^{t r} \boldsymbol{x}_{/ r}> \\
=-<\boldsymbol{N}, \nabla^{I I I}(R, N)-R \boldsymbol{N}> \\
-<\boldsymbol{x}, 2 \boldsymbol{N}>-2 e^{i k}<\boldsymbol{x}_{/ i}, b_{k t} g^{t r} \boldsymbol{x}_{/ r}> \\
=2 W-R .
\end{gathered}
$$

We consider now the surface $M$ of finite $I I I$-type 1. Then we have $\Delta^{I I I} \boldsymbol{x}=k \boldsymbol{x}$, where $k$ is a constant eigenvalue.

From $(15)$ we get $\nabla^{I I I}(R, N)-R \boldsymbol{N}=k \boldsymbol{x}$. Taking the inner product of both sides of this equation with $N$ we find $R=k W$. From the formula (16) we find that

$$
\Delta^{I I I} W=(2-k) W, \Delta^{I I I} R=(2-k) R .
$$

Thus, we have proved the following:

Theorem 3. Let $M$ be a surface in $E^{3}$ of finite IIItype 1 with corresponding eigenvalue $l$. Then the support function $W$ and the sum of the principal radii of curvature $R$ are of eigenfunctions of the Laplacian $\Delta^{I I I}$ with corresponding eigenvalue $2-k$.

Let now $M$ be a minimal surface. Then we have

$$
R=\frac{2 H}{K}=0 \text {. }
$$

Thus from the equation (16) we get $\Delta^{I I I} W=2 W$. So we have

Corollary 1. Let $M$ be a minimal surface. Then the support function $W$ is of an eigenfunction of $\Delta^{\text {III }}$ with corresponding eigenvalue equal 2.

We consider now a surface $M$ which is defined on a simply connected domain $D$ in the $(u, v)$-plane with parametric representation $M: \boldsymbol{y}=\boldsymbol{y}(u, v)$. Let $M^{*}$ be a parallel surface of $M$ in distance $\lambda=$ const. $\neq 0$, so that

$$
1-2 \lambda H+\lambda^{2} K \neq 0 .
$$

Then $M^{*}$ possesses the position vector 


$$
y^{*}=y+\lambda N .
$$

We represent by $K^{*}$ and $H^{*}$ the Gauss and mean curvature of $M^{*}$ respectively, It's well-known that

$$
\begin{aligned}
K^{*} & =\frac{K}{1-2 \lambda H+\lambda^{2} K}, \\
H^{*} & =\frac{H-\lambda K}{1-2 \lambda H+\lambda^{2} K} .
\end{aligned}
$$

Thus we obtain

$$
R^{*}=\frac{2 H^{*}}{K^{*}}=R-2 \lambda
$$

Besides, the surfaces $M, M^{*}$ have same unit normal vector $N$. Hence $I I I=I I I^{*}$ and $\Delta^{I I I}=\Delta^{I I I^{*}}$, which we will denote it, for simplicity, by $\Delta$. We mention the following theorem which was proved in [23]

Theorem 4. Let $M$ be a surface satisfying $\frac{H}{K}=\mu=$ const. $\neq 0$, which is not part of a sphere. Then $M$ is of null III-type 2. use.

We prove now the following theorem for later

Theorem 5. The surface $M^{*}$ is a parallel surface of the minimal $M$ in $E^{3}$ if and only if the sum of the principal radii of curvature $R^{*}$ of $M^{*}$ is constant.

Proof. Let $M: \boldsymbol{y}=\boldsymbol{y}(u, v)$, defined on the domain $D$, be a minimal surface in $E^{3}$, and

$$
M^{*}: \boldsymbol{y}^{*}=\boldsymbol{y}+\lambda N, \lambda \neq 0
$$

being a parallel surface of $M$. Taking into account relation (17) and that $H=0$, we get $R^{*}=-2 \lambda=$ const.. Thus we proved the first part of the theorem.

Conversely, let $R^{*}=$ const. $\neq 0$. Then from Theorem 4, $M^{*}$ is of null III- type 2. Therefore from (1) there exist nonconstant eigenvectors $\boldsymbol{y}_{1}(u, v)$ and $\boldsymbol{y}_{2}(u, v)$ defined on the same domain $D$ such that

$$
\boldsymbol{y}^{*}=\boldsymbol{y}_{1}+\boldsymbol{y}_{2}
$$

where $\Delta \boldsymbol{y}_{1}=\mu_{1} \boldsymbol{y}_{1}, \Delta \boldsymbol{y}_{2}=\mu_{2} \boldsymbol{y}$, also since $M^{*}$ is of null III- type 2, we have $\mu_{1}=0$.

From $\Delta \boldsymbol{y}^{*}=\Delta \boldsymbol{y}_{1}+\Delta \boldsymbol{y}_{2}$, we get

$$
\Delta \boldsymbol{y}^{*}=\mu_{2} \boldsymbol{y}_{2}
$$

Besides, since $R^{*}=$ const. $\neq 0$, we find

$$
\Delta y^{*}=-R^{*} N \text {. }
$$

Thus from (19) and (20), one finds

$$
\mu_{2} \boldsymbol{y}_{2}=-R^{*} N
$$

or $\boldsymbol{y}_{2}=c N$, where $\mathrm{c}=-\frac{R^{*}}{\mu_{2}}$, and then (18) becomes

$$
\boldsymbol{y}^{*}=\boldsymbol{y}_{1}+c \boldsymbol{N} \text {. }
$$

The differential of the above equation is

$$
d \boldsymbol{y}^{*}=d \boldsymbol{y}_{1}+c d \boldsymbol{N} .
$$

Taking the inner product of both sides of (22) with $N$ yields

$$
<d \boldsymbol{y}_{1}, \boldsymbol{N}>=0 .
$$

If we prove that

$$
\boldsymbol{y}_{1 / \mathrm{u}} \times \boldsymbol{y}_{1 / \mathrm{v}} \neq \mathbf{0}, \quad \forall(u, v) \in D,
$$

where $\times$ is the Euclidean cross product, then $\boldsymbol{y}_{1}(u, v)$ is a regular parametric representation of a surface in $E^{3}$. We have

$$
\boldsymbol{y}_{1}=\boldsymbol{y}^{*}-\lambda \boldsymbol{N}^{*}
$$

Using the Weingarten equations

$$
\boldsymbol{N}_{/ i}=-b_{i j} g^{j k} \boldsymbol{y}^{*} / k \text {. }
$$

and the equation (24), it follows that

$$
\begin{gathered}
\boldsymbol{y}_{1 / \mathrm{u}} \times \boldsymbol{y}_{1 / \mathrm{v}}=\left(\boldsymbol{y}_{/ \mathrm{u}}-\lambda \boldsymbol{N}_{/ \mathrm{u}}\right) \times\left(\boldsymbol{y}_{/ \mathrm{v}}^{*}-\lambda \boldsymbol{N}_{/ \mathrm{v}}\right) \\
=\left(\boldsymbol{y}_{/ \mathrm{u}}^{*} \times \boldsymbol{y}_{/ \mathrm{v}}^{*}\right)-\lambda\left(\boldsymbol{y}_{/ \mathrm{u} \times}^{*} \times \boldsymbol{N}_{/ 2}\right)+\lambda\left(\boldsymbol{y}_{/ \mathrm{v}}^{*} \times \boldsymbol{N}_{/ \mathrm{u}}\right) \\
+\lambda^{2}\left(\boldsymbol{N}_{/ \mathrm{u}} \times \boldsymbol{N}_{/ \mathrm{v}}\right) \\
=\left(1-2 \lambda H+\lambda^{2} K\right)\left(\boldsymbol{y}_{/ \mathrm{u}}^{*} \times \boldsymbol{y}_{/ \mathrm{v}}\right) \neq \mathbf{0}, \\
\forall(u, v) \in D .
\end{gathered}
$$

Hence, on account of (23) and (25), we conclude that $\boldsymbol{y}_{1}(u, v)$ is a regular parametric representation of a surface in $E^{3}$ with $N$ its Gauss map.

Since $\Delta \boldsymbol{y}_{1}=\mathbf{0}$. Consequently, from Theorem 1, $\boldsymbol{y}_{1}(u, v)$ is a minimal surface. Thus from (21), we obtain that $M^{*}$ is a parallel surface of a minimal. Now we mention and prove our main theorem. 
Theorem 6. The only surfaces of revolution in $E^{3}$ of which the sum of the radii of the principal curvature $R$ is constant are parts of spheres which are of finite III-type 1, catenoids which are of finite null III-type 1 , and the parallel surfaces to the catenoids, which are of finite null III-type 2.

\section{Proof of main result}

Let $C$ be the profile curve of a surface of revolution $M$ of the differentiation class $C^{3}$. We suppose that $C$ lies on the $x z$-plane, the axis of revolution of $M$ is the $z$-axis and $C$ is parametrized by it's arc-length $s$. Then $C$ admits the parametric representation

$$
\boldsymbol{r}(s)=(f(s), 0, g(s)), s \in J,(J \subset \Re),
$$

where $f, g$ are smooth functions and $f$ is a positive function. Then the position vector of $M$ is given by see $([14,24])$

$$
\begin{gathered}
\boldsymbol{y}(s, v)=(f(s) \cos v, f(s) \sin v, g(s)), \\
s \in J, v \in[0,2 \pi) .
\end{gathered}
$$

Since $s$ is the arc-length of $C$, then we have

$$
\left(f^{\prime}\right)^{2}+\left(g^{\prime}\right)^{2}=1
$$

where ${ }^{\prime}:=\frac{d}{d s}$. Furthermore if $f^{\prime} g^{\prime}=0$, then $f=$ const. or $g=$ const. and $M$ would be a circular cylinder or part of a plane, respectively. A case that has been excluded since $M$ would consist only of parabolic points.

Denoting by $\kappa$ the curvature of the curve $C$ and $r_{1}, r_{2}$ the principal radii of curvature of $M$, we have

$$
r_{1}=\frac{1}{\kappa}, \quad r_{2}=\frac{f}{g^{\prime}},
$$
is

The parametric representation of the Gauss map $N$

$$
N(s, v)=\left(-g^{\prime} \cos v,-g^{\prime} \sin v,-f^{\prime}\right) .
$$

Now, by using the natural frame $\left\{\boldsymbol{N}_{s}, \boldsymbol{N}_{v}\right\}$ of $M$ defined by

and

$$
\boldsymbol{N}_{s}=\left(-g^{\prime \prime} \cos v,-g^{\prime \prime} \sin v, f^{\prime \prime}\right)
$$

$$
N_{v}=\left(g^{\prime} \sin v,-g^{\prime} \cos v, 0\right)
$$

the components $e_{i j}$ of the third fundamental form in (local) coordinates are the following

$$
\begin{gathered}
e_{11}=<\boldsymbol{N}_{s}, \boldsymbol{N}_{u}>=\left(g^{\prime \prime}\right)^{2}+\left(f^{\prime \prime}\right)^{2}, \\
e_{12}=<\boldsymbol{N}_{s}, \boldsymbol{N}_{v}>=0, \mathrm{e}_{22}=<\boldsymbol{N}_{v}, \boldsymbol{N}_{v}>=\left(g^{\prime}\right)^{2} .
\end{gathered}
$$

The Beltrami operator $\Delta$ in terms of local coordinates $(s, v)$ with respect to the third fundamental form of $S$ can be expressed as follows

$$
\Delta=-\frac{1}{\kappa^{2}} \frac{\partial^{2}}{\partial s^{2}}+\frac{g^{\prime} \kappa^{\prime}-\kappa g^{\prime \prime}}{g^{\prime} \kappa^{3}} \frac{\partial}{\partial s}-\frac{1}{g^{\prime 2}} \frac{\partial^{2}}{\partial v^{2}} .
$$

On account of (27) we put

$$
f^{\prime}=\cos \varphi, g^{\prime}=\sin \varphi,
$$

where $\varphi=\varphi(s)$. Then $\kappa=\varphi^{\prime}$ and the parametric representation (28) of the unit vector $N$ of $M$ becomes

$$
N(s, v)=\{-\sin \varphi \cos v,-\sin \varphi \sin v, \cos \varphi\} .
$$

Also relation (29) takes the following form

$$
\Delta=-\frac{1}{\varphi^{\prime 2}} \frac{\partial^{2}}{\partial u^{2}}+\left(\frac{\varphi^{\prime \prime}}{\varphi^{\prime 3}}-\frac{\cos \varphi}{\varphi^{\prime} \sin \varphi}\right) \frac{\partial}{\partial u}-\frac{1}{\sin ^{2} \varphi} \frac{\partial^{2}}{\partial v^{2}} .
$$

For the sum of the principal radii of curvature $R$ $=r_{1}+r_{2}=\frac{2 H}{K}$, one finds

$$
R=\frac{f}{\sin \varphi}+\frac{1}{\varphi^{\prime}}
$$

Taking the derivative of (32) we find

$$
R^{\prime}=-\frac{\varphi^{\prime \prime}}{\varphi^{\prime 2}}-\frac{f \varphi^{\prime} \cos \varphi}{\sin ^{2} \varphi}+\frac{\cos \varphi}{\sin \varphi} .
$$

Let $\left(y_{1}, y_{2}, y_{3}\right)$ be the coordinate functions of (26). By virtue of (31), we obtain

$$
\begin{gathered}
\Delta y_{1}=\left(\frac{\varphi^{\prime \prime} \cos \varphi}{\varphi^{\prime 3}}-\frac{1}{\varphi^{\prime} \sin \varphi}+\frac{2 \sin \varphi}{\varphi^{\prime}}+\frac{f}{\sin ^{2} \varphi}\right) \cos v \\
\Delta y_{2}=\left(\frac{\varphi^{\prime \prime} \cos \varphi}{\varphi^{\prime 3}}-\frac{1}{\varphi^{\prime} \sin \varphi}+\frac{2 \sin \varphi}{\varphi^{\prime}}+\frac{f}{\sin ^{2} \varphi}\right) \sin v \\
\Delta y_{3}=-\frac{2 \cos \varphi}{\varphi^{\prime}}+\frac{\varphi^{\prime \prime} \sin \varphi}{\varphi^{\prime 3}} .
\end{gathered}
$$

From (32) and (33), equations (34), (35) and (36) become respectively 


$$
\begin{aligned}
& \Delta y_{1}=\left(R \sin \varphi-\frac{R^{\prime} \cos \varphi}{\varphi^{\prime}}\right) \cos v, \\
& \Delta y_{2}=\left(R \sin \varphi-\frac{R^{\prime} \cos \varphi}{\varphi^{\prime}}\right) \sin \nu, \\
& \Delta y_{3}=-\frac{R^{\prime} \sin \varphi}{\varphi^{\prime}}-R \cos \varphi .
\end{aligned}
$$

We obtain the following two cases:

Case $I$. $R \equiv 0$. Thus $H \equiv 0$. Consequently $M$, being a minimal surface of revolution, is a catenoid.

Case II. $R=$ const. $\neq 0$. From (37), (38), and (39) we obtain

$$
\left.\begin{array}{c}
\Delta y_{1}=R \sin \varphi \cos v \\
\Delta y_{2}=R \sin \varphi \sin v \\
\Delta y_{3}=-R \cos \varphi
\end{array}\right\}
$$

Let $\left(N_{1}, N_{2}, N_{3}\right)$ be the coordinate functions of $\boldsymbol{N}$. From (29), relations (40) can be written

$$
\Delta y_{1}=-R N_{1}, \Delta y_{2}=-R N_{2}, \Delta y_{3}=-R N_{3} \text {, }
$$

and hence

$$
\Delta y=-R N .
$$

In view of (7) and (41) we have

$$
\Delta^{m} \boldsymbol{y}=-\left(2^{m-1}\right) R \boldsymbol{N}
$$

Now, if $M$ is of finite type $k$, then there exist real numbers $c_{1}, c_{2}, \ldots, c_{k}$ such that

$$
\Delta^{k} \boldsymbol{y}+c_{1} \Delta^{k-1} \boldsymbol{y}+\ldots+c_{k} \boldsymbol{y}=\mathbf{0} .
$$

From (41) and (42) relation (43) becomes

$$
\begin{gathered}
-2^{\mathrm{k}-1} R \boldsymbol{N}-2^{\mathrm{k}-2} c_{l} R \boldsymbol{N}-\ldots-c_{k-l} R \boldsymbol{N}+c_{k} \boldsymbol{y}=\mathbf{0}, \\
\text { or } \quad c \boldsymbol{N}+c_{k} \boldsymbol{y}=\mathbf{0},
\end{gathered}
$$

where $\mathrm{c}=-R\left(2^{\mathrm{k}-1}+2^{\mathrm{k}-2} c_{1}+\ldots+c_{k-1}\right)=$ const.

On one hand, if $c_{\kappa} \neq 0$, then from (44) we find $\boldsymbol{y}=-$ $\frac{c}{c_{k}} \boldsymbol{N}$, thus we get $|\boldsymbol{y}|=\left|\frac{c}{c_{k}}\right|$ and so $M$ is a sphere.

From Theorem 2, $M$ is of finite III-type 1 . On the other hand, if $c_{k}=0$, then $M$ is of null type $k$. Because of $R=$ const., and according to Theorem 4, and Theorem 5, $M$ is of null III-type 2 which is a parallel surface of a minimal.

\section{References:}

[1] H. Al-Zoubi, S. Stamatakis, Ruled and Quadric surfaces satisfying $\Delta^{I I I} \boldsymbol{x}=A \boldsymbol{x}, J$. for Geom. and Graphics, 20 (2016), pp.147-157.

[2] H. Al-Zoubi, S. Stamatakis, W. Al Mashaleh and M. Awadallah, Translation surfaces of coordinate finite type, Indian J. of Math. 59 (2017), pp. 227-241.

[3] H. Al-Zoubi, Tubes of finite II-type in the Euclidean 3-space, WSEAS Trans. Math. 17 (2018), pp. 1-5.

[4] H. Al-Zoubi, S. Al-Zubi, S. Stamatakis and H. Almimi, Ruled surfaces of finite Chen-type. $J$. Geom. and Graphics 22 (2018), pp. 15-20.

[5] H. Al-Zoubi, K. M. Jaber, S. Stamatakis, Tubes of finite Chen-type, Commun. Korean Math. Soc. 33 (2018), pp. 581-590.

[6] H. Al-Zoubi, M. Al-Sabbagh, S. Stamatakis, On surfaces of finite Chen III-type, Bull. Belgian Math. Soc. 26 (2019), pp. 177-187.

[7] H. Al-Zoubi, A. Dababneh, M. Al-Sabbagh, Ruled surfaces of finite II-type, WSEAS Trans. Math. 18 (2019), pp. 1-5.

[8] H. Al-Zoubi, T. Hamadneh, M. Abu Hammad, and M. Al-Sabbagh, Tubular surfaces of finite type Gauss map, J. Geom. Graph. 25 (2021), pp. 45-52.

[9] H. Al-Zoubi, M. Al-Sabbagh, Anchor rings of finite type Gauss map in the Euclidean 3-space, Int. J. Math. Comput. Methods 5 (2020), 9-13.

[10] H. Al-Zoubi, H. Alzaareer, T. Hamadneh, M. Al Rawajbeh, Tubes of coordinate finite type Gauss map in the Euclidean 3-space, Indian J. Math. 62 (2020), 171-182.

[11] H. Al-Zoubi, T. Hamadneh, Quadric surfaces of coordinate finite type Gauss map, arXiv 2006.04529v1, May (2020).

[12] H. Al-Zoubi, W. Al Mashaleh Surfaces of finite type with respect to the third fundamental form, Int. Conf. Elect. Eng. Inf. Tech. (JEEIT) Jordan (2019).

[13] H. Al-Zoubi, T. Hamadneh, H. Alzaareer, M. Al-Sabbagh, Tubes in the Euclidean 3-space with coordinate finite type Gauss map, Int. Conf. Inf. Tech. (ICIT) Jordan (2021).

[14] H. Al-Zoubi, Surfaces of finite III-type, Int. J. Math. Models and Methods in Applied Sciences 15 (2021), 190-194.

[15] B.-Y. Chen, Some open problems and conjectures on submanifolds of finite type, Soochow J. Math. 17 (1991), pp. 169-188.

[16] B.-Y. Chen, Total mean curvature and submanifolds of finite type, Second edition, World Scientific Publisher, (2014). 
[17] B.-Y. Chen, A report on submanifolds of finite type, Soochow J. Math. 22 (1996), pp. 117-337.

[18] F. Dillen, J. Pas, and L. Verstralen, On the Gauss map of surfaces of revolution, Bull. Inst. Math. Acad. Sinica 18 (1990), no. 3, pp. 239246.

[19] H. Huck, U. Simon, R. Roitzsch, W. Vortisch, R. Walden, B. Wegner, and W. Wendl, Beweismethoden der Di_erentialgeometrie im Grossen, Lecture Notes in Mathematics. 335 (1973).

[20] Y. H. Kim, C. W. Lee, and D. W. Yoon, On the Gauss map of surfaces of revolution without parabolic points, Bull. Korean Math. Soc. 46 No. 6, (2009), pp. 1141-1149.

[21] M. Mhailan, M. Abu Hammad, M. Al Horani, R. Khalil, On fractional vector analysis, $J$. Math. Comput. Sci. 10 (2020), 2320-2326.

[22] B. Senoussi, H. Al-Zoubi, Translation surfaces of finite type in $\mathrm{Sol}_{3}$, Comment. Math. Univ. Carolin. 61 (2020), pp. 237-256.

[23] S. Stamatakis, H. Al-Zoubi, On surfaces of finite Chen-type, Results. Math., 43 (2003), pp. 181-190.

[24] S. Stamatakis, H. Al-Zoubi, Surfaces of revolution satisfying $\Delta^{I I I} \boldsymbol{x}=\mathrm{A} \boldsymbol{x}, \mathrm{J}$. for Geom. and Graphics, 14 (2010), pp. 181-186.

\section{Contribution of individual authors to the creation of a scientific article (ghostwriting policy)}

Hassan Al-Zoubi carried out the introduction and the main result of the article.

Farhan Abdel-Fattah has improved section 2.

Mutaz Al-Sabbagh has reviewed and checked the calculations of Theorem 2 and Theorem 3.

\section{Sources of funding for research presented in a scientific article or scientific article itself}

This study did not receive any funding in any form.

\section{Creative Commons Attribution \\ License 4.0 (Attribution 4.0 \\ International , CC BY 4.0)}

This article is published under the terms of the Creative Commons Attribution License 4.0 https://creativecommons.org/licenses/by/4.0/deed.en $\underline{\text { US }}$ 